\title{
Diffusion Tensor and Functional MRI Fusion with Anatomical MRI for Image-Guided Neurosurgery
}

\author{
Ion-Florin Talos $^{1,2}$, Lauren O’Donnell ${ }^{1,3}$, Carl-Fredrick Westin ${ }^{1}$, Simon K. Warfield ${ }^{1}$, \\ William Wells III', Seung-Schik Yoo ${ }^{1}$, Lawrence P. Panych ${ }^{1}$, Alexandra Golby ${ }^{2}$, \\ Hatsuho Mamata ${ }^{1}$, Stefan S. Maier ${ }^{1}$, Peter Ratiu ${ }^{1}$, Charles R.G. Guttmann ${ }^{1}$, \\ Peter M. Black ${ }^{2}$, Ferenc A. Jolesz ${ }^{1}$, and Ron Kikinis ${ }^{1}$ \\ ${ }^{1}$ Department of Radiology \\ ${ }^{2}$ Division of Neurosurgery, Brigham and Women's Hospital, Harvard Medical School \\ Boston MA, USA \\ ${ }^{3}$ Artificial Intelligence Laboratory, Massachusetts Institute of Technology \\ Cambridge MA, USA \\ talos@bwh. harvard. edu
}

\begin{abstract}
In order to achieve its main goal of maximal tumor removal while avoiding postoperative neurologic deficits, neuro-oncological surgery is strongly dependent on image guidance. Among all currently available imaging modalities, MRI provides the best anatomic detail and is highly sensitive for intracranial pathology. However, conventional MRI does not detect the exact location of white matter tracts or areas of cortical activation. This essential information can be obtained non-invasively by means of diffusion tensor MRI (DT-MRI) and functional MRI (fMRI) respectively. Here we present our initial experience with fMRI and DT-MRI for surgical planning and guidance in ten brain tumor cases.
\end{abstract}

\section{Introduction}

Data supporting the thesis that gross tumor removal results in prolonged overall and recurrence-free survival is accumulating [1], hence the main goal of neurooncological surgery is maximal tumor removal while minimizing post-operative neurological deficits. However, for a multitude of reasons, achieving this goal may be challenging: 1) many intra-axial tumors, such as low-grade glial neoplasms, may remain, at least in part, occult to visual inspection; 2) the brain alters its shape in response to surgical manipulation and anesthesia (brain shift)[2], making tumor localization even more difficult; 3 ) neither visual inspection, nor conventional imaging can provide accurate information about the relationship between tumor and functionally important cortical and white matter areas.

CT and MRI have a much higher sensitivity than the human eye in detecting intraaxial tumors. The use of CT or MRI acquired in advance of surgery along with neuronavigation systems has become routine at most institutions. However, the use of preoperatively acquired images for surgical guidance is strongly limited by the intraoperative shifting of the brain. Introduced into clinical practice in the mid 1990s, intraoperative MRI (iMRI) combines the high sensitivity in detecting the tumor with updated images, capable to compensate for brain shift changes and monitor the resec- 
tion progress [3]. Most currently available iMRI systems are integrated with neuronavigation systems.

While conventional MRI faithfully describes cortical surface, deep gray matter and CSF space anatomy, it does not provide insight into the complex organization of the cerebral white matter, nor does it provide functional information. By means of fMRI, location and extent of activated cortical areas in response to sensory stimulation and motor or memory tasks can readily be detected, by taking advantage of variations in local blood flow and oxygenation. DT-MRI provides information about location, direction and extent of white matter fiber tracts. Tracking white matter tracts is based on MRI-detection of molecular motion of tissue water. In highly structured tissue, such as cerebral white matter, water molecule diffusion is restricted in the direction perpendicular to the fibers, due to cell membranes and myelin sheaths, whereas it is relatively unrestricted in the direction parallel to the fibers, i.e. it shows an anisotropic pattern. The water molecule diffusion within a voxel can be conceptualized as an ellipsoid shaped tensor. The directions of the three main axes of the ellipsoid represent the eigenvectors, their magnitude the eigenvalue of the tensor.

The impact of intrinsic brain tumors on cortical gray matter has been extensively studied in vivo by means of fMRI [4], magneto-encephalography (MEG) [5] and direct electrical stimulation (ECS) [6]. These studies have clearly demonstrated that intrinsic brain tumors grow by infiltration of surrounding brain parenchyma; functionally important areas can reside within grossly abnormal tissue. It follows therefore, that post-operative neurological deficits may occur even if the surgical manipulation is strictly limited to the area of abnormality seen on MRI.

In vivo studies on the impact of intrinsic brain tumors on white matter tracts are nowadays possible by means of DT-MRI. The preliminary experience accumulated by our group, as well as others, indicates that morphologically preserved white matter tracts can be found within the tumor boundaries. Besides its scientific consequences, this fact has immediate relevance for the surgical treatment of brain tumors.

Intraoperative acquisition of DT-MRI and fMRI is not practical, due to long scanning and image processing times. On the other side, the use of image data acquired in advance for neuronavigation is limited by brain shift. One possible solution to this problem is the use of robust biomechanical simulation algorithms combined with intraoperative conventional MRI [7], in order to preserve the accuracy of preoperatively acquired fMRI and DT-MRI.

The aim of the present study was to evaluate the usefulness of fMRI and DT-MRI for assessing the impact of intrinsic brain tumors on cerebral gray and white matter and the potential use of these new imaging modalities for surgical guidance.

\subsection{Patient Population, Data Acquisition, and Processing}

10 patients (Table 1) with intraaxial supratentorial tumors were selected for this study. Based on previous MRI exams, it was estimated that these lesions may be located in close proximity to "eloquent" cortical areas and important white matter fiber tracts, such as the primary and supplementary motor area, Brocca and Wernicke's speech areas, cortico-spinal tract, optic radiation, superior longitudinal fasciculus, uncinate fasciculus. 
Table 1.

\begin{tabular}{|c|c|c|c|c|}
\hline $\begin{array}{l}\text { Case } \\
\text { No. }\end{array}$ & $\begin{array}{l}\text { Sex,Age } \\
\text { (yrs.) }\end{array}$ & Tumor location & Histopathology & $\begin{array}{l}\text { Eloquent cortical and white mat- } \\
\text { ter areas affected }\end{array}$ \\
\hline 1 & F 33 & $\mathrm{~L}$ frontal & suspicious for LGG & SMA, motor strip, motor pathway \\
\hline 2 & F 34 & R temporal & $\begin{array}{l}\text { oligodendroglioma } \\
\text { WHO II }\end{array}$ & Wernicke's area, optic radiation \\
\hline 3 & F 62 & $\mathrm{~L}$ frontal & $\begin{array}{l}\text { oligodendroglioma } \\
\text { WHO II }\end{array}$ & SMA, motor pathway \\
\hline 4 & M 62 & $\mathrm{R}$ frontal medial & $\begin{array}{l}\text { anaplasic astrocy- } \\
\text { toma (WHO III) }\end{array}$ & Motor strip, motor pathway \\
\hline 5 & M 38 & $\mathrm{~L}$ frontal & $\begin{array}{l}\text { astrocytoma } \\
\text { WHO II }\end{array}$ & Motor strip, motor pathway \\
\hline 6 & F 45 & $\mathrm{R}$ fronto-parietal & $\begin{array}{l}\text { anaplastic astrocy- } \\
\text { toma (WHO III) }\end{array}$ & $\begin{array}{l}\text { Motor and sensory strip, motor } \\
\text { pathway, arcuate (superior longitu- } \\
\text { dinal) fasciculus }\end{array}$ \\
\hline 7 & F 46 & R occipital & $\begin{array}{l}\text { oligodendroglioma } \\
\text { WHO II }\end{array}$ & Optic radiation \\
\hline 8 & M 23 & $\mathrm{R}$ insular & ganglioglioma & Motor pathway, uncinate fasciculus \\
\hline 9 & F 18 & $\mathrm{R}$ frontal & $\begin{array}{l}\text { anaplastic astrocy- } \\
\text { toma }\end{array}$ & Motor strip, motor pathway \\
\hline 10 & M 49 & L frontal & $\begin{array}{l}\text { oligodendroglioma } \\
\text { WHO II }\end{array}$ & $\begin{array}{l}\text { SMA, motor pathway, corpus callo- } \\
\text { sum }\end{array}$ \\
\hline
\end{tabular}

\subsection{Imaging Protocol}

After informed consent, the patients underwent the following MR imaging protocol on a 1.5T Horizon Echospeed 5.6 scanner (General Electric, Milwaukee, MN) a few days ahead of the scheduled surgery:

a) Anatomic Imaging: (1) whole brain sagittal 3D-SPGR (slice thickness $1.5 \mathrm{~mm}$, $\mathrm{TE} / \mathrm{TR}=6 / 35 \mathrm{msec}, \mathrm{FA}=75^{\circ}, \mathrm{FOV}=24 \mathrm{~cm}$, matrix=256x256); (2) axial T2-weighted fast-spin-echo (slice thickness 5mm, TE/TR 100/3000 msec, FOV=22cm, matrix $=256 x 192)$, and (3) phase-contrast MR angiography $(1.5 \mathrm{~mm}$ sagittal slices, $\mathrm{TR}=32$ $\mathrm{msec}, \mathrm{FA}=20^{\circ}, \mathrm{FOV}=24 \mathrm{cmm}$, matrix $=256 \times 128$, Venc $\left.=60\right)$. Pre and post-contrast $\mathrm{T} 1-$ weighted spin echo images were also acquired to detect enhancing tumor tissue.

b) Functional MRI: Reference dual gradient echo $(\mathrm{TE} 1 / \mathrm{TE} 2 / \mathrm{TR}=\mathrm{MIN} / 50 /$ $2500 \mathrm{msec}, 128 \times 64$ matrix) and T1-weighted SPGR (TE/TR $=\mathrm{MIN} / 30, \mathrm{FA}=30^{\circ}$, $256 \times 128$ ) images were acquired through the same region as the planned echo-planar (EPI) fMRI scans. The reference images were subsequently used for rigid registration with the high-resolution 3D-SPGR series acquired in the anatomical imaging session. For each fMRI run, a set of $4 \mathrm{~mm}$ thick EPI slices through the region of interest, covering the tumor and the potential surgical corridors were acquired with the following parameters: TR/TE $=2000 / 50 \mathrm{msec}, F A=90, F O V=24 \mathrm{~cm}$ and matrix $=64 \times 64$. The voxel size was $3.75 \times 3.75 \times 4 \mathrm{~mm}^{3}$. For mapping motor areas, a fist clenching paradigm was administered at a pace of $1 \mathrm{~Hz}$. For mapping language areas, a semantic language task was employed (Howard 2001). For visual mapping, light stimuli were presented to both eyes simultaneously at a frequency of $1 \mathrm{~Hz}$. Irrespective of the task paradigm, five task epochs of 30 seconds duration were interleaved with six 30second rest epochs. The auditory cues were administered using the Presentation software (Neurobehavioral Systems, CA). 
c) Diffusion Tensor Imaging: axial and coronal line scan diffusion images (LSDI) ( $\mathrm{TE}=64 \mathrm{msec}, \mathrm{TR}=2592 \mathrm{msec}$, slice thickness $4 \mathrm{~mm}$, slice gap $1 \mathrm{~mm}$ were acquired, covering the entire region of interest as well as "landmark" regions, i.e. areas where the relevant fiber tracts show high density (e.g. ventral brain stem for the corticospinal tract, lateral geniculate body for the optic radiation).

d) Optionally, MR-Spectroscopy (PRESS) from the tumor was also acquired.

\subsection{Image Processing}

a) Anatomic Data: brain and ventricular system segmentations were obtained from the 3D-SPGR, using a curved surface evolution algorithm; the tumors were segmented manually from either T2-FSE (non-enhancing lesions) or post-contrast 3DSPGR (enhancing lesions). From the resulting label maps, 3D-models were generated in the 3D-Slicer [8] (Figure 2).

b) Functional MRI: SPM99 was used for reconstruction and motion correction of the EPI data sets. In house software was used to calculate pixel-by-pixel paired $t$-test scores across the time course of the EPI acquisitions, which were then converted to corresponding $p$-values. In the next step, the fMRI data was rigidly registered to the high-resolution 3D-SPGR [9]. The 3D-Slicer was then used for thresholding $\left(\mathrm{p}<10^{-6}\right)$ and 3D model generation from the activation maps (Figures 2 and 4).

c) Diffusion Tensor Data: Currently, several fiber tract visualization methods are available: headless arrows to represent the in-plane component of the principal eigenvector, along with a color-coded out-of-plane component [10], three-dimensional tractography $[11,12]$ etc.. For the present study, we have used the 3D tractography method described by Westin et al. [12]. This algorithm is fully implemented in the 3D-Slicer software package. The baseline acquisition of the LSDI series was used for registering the LSDI dataset to the high-resolution 3D-SPGR. For initialization of the 3D tractography algorithm, seeding points were placed in the landmark areas. Alternatively, seeding points were placed in the subcortical white matter subjacent to areas of cortical activation seen on fMRI. The 3D-tractography results were checked, in every case, against results obtained using Peled's 2D tractography method (Figures 1 and 3).

\subsection{Non-rigid Registration}

It is now a widely acknowledged fact that the brain alters its shape during surgery due to CSF loss, swelling, pharmacological effects, variations of vascular tone and tissue hydration state, tumor removal. These changes occur according to a non-linear pattern [2]. In order to maintain the accuracy of preoperatively acquired images, a mechanism of compensating for these changes is indispensable. Our method of tracking volumetric brain deformation during MRI-guided neurosurgical procedures has been previously described [7]. Briefly, intraoperative MR image updates provide information on the changes in brain shape and position relative to the preoperative scans. Brain and lateral ventricles surface changes are used to track the volumetric deformation field, which is then inferred to preoperative data sets, such as functional MRI and 3Dtractography, in order to warp them into the configuration of the deformed brain using a finite element biomechanical model. The parallel algorithm is executed on a SunFire 
6800 symmetric multiprocessor machine with $12750 \mathrm{MHz}$ UltraSPARC III CPU's and 12 GB of RAM in 5-7 minutes. This short time is fully compatible with the constraints imposed by the ongoing surgery.

\section{Results}

The fusion of functional, DT- and anatomical MRI data was successful in all cases. In five cases, functional MRI has been successfully used for surgical navigation. The fMRI activation maps could be directly compared with the responses elicited by cortical stimulation (ECS). fMRI faithfully predicted the location of motor and speech areas and this fact could be confirmed by means of direct cortical stimulation (Figure 4). The information provided by fMRI was considered useful for surgical guidance.

In our small patient series, morphologically preserved fiber tracts were identified in all cases, irrespective of histopathological grading, even in areas of contrast enhancement, generally considered to display a more aggressive biological behavior (Figure 3). Besides infiltration, fiber tract displacement could also be observed even in the same tumor (Figure 1).

\subsection{Illustrative Cases}

All patients underwent preoperative imaging according to the imaging protocol described in the previous section. In all cases, the surgical procedure was carried out with intraoperative MRI-guidance.

\section{Case 1 (Figure1)}

This case of left frontal oligodendroglioma WHO II/IV in a 62 year old female illustrates multiple white matter changes induced by the same tumor. While the posterior part of the tumor appears to displace the white matter tracts, corpus callosum projection fibers as well as descending (motor) fibers are clearly identifiable in the anterior part of the tumor. Since the fMRI identified the origin of the descending fibers in the supplementary motor area (SMA), it was decided to proceed aggressively and to attempt gross total tumor resection. Pos-surgery, the patient developed a reversible SMA-syndrome (dysphasia and contralateral hemiplegia).

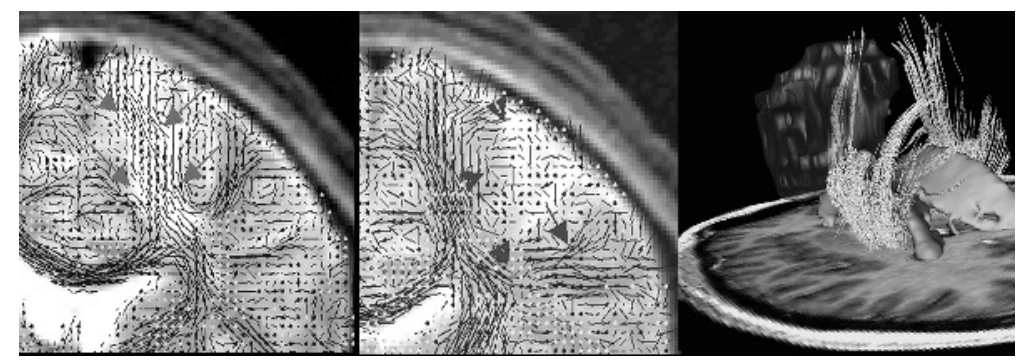

Fig. 1. (case 1). Left frontal oligodendroglioma WHO II/IV. Anterior (left) and posterior coronal view (middle). Right: 3D-tractography) (see text). 


\section{Case 2 (Figure 2)}

This 23 year old left handed male presented with incoordination and weakness of his left hand. The MRI showed a mass lesion in the right temporal lobe (histology: ganglioglioma), extending superiorly into the posterior limb of the internal capsule. Although the conventional MRI suggested tumor compression of the right optic tract and right cerebral peduncle, the DT-tractography clearly demonstrated cortico-spinal fibers traversing the medial portion of the tumor. The majority of the tumor was removed, with a small medial remnant, in order to preserve the motor fibers. No postoperative neurologic deficit.
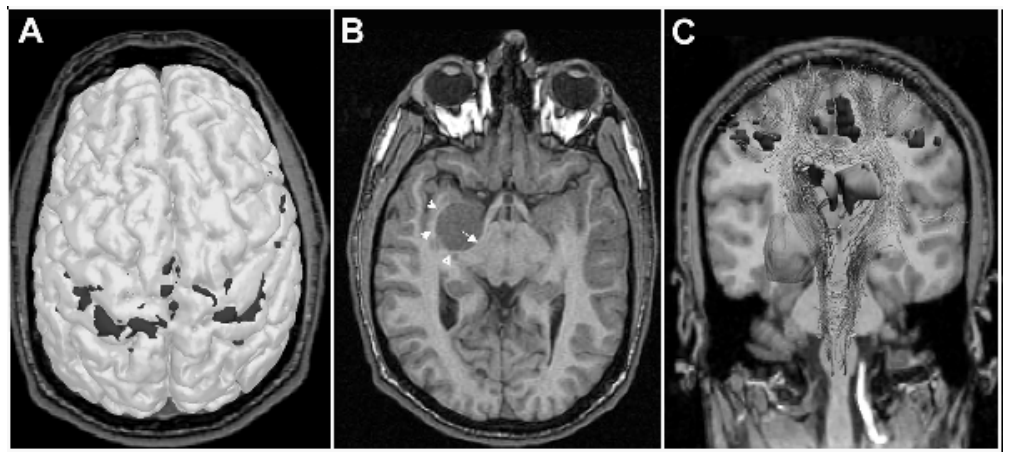

Fig. 2. (case 2). Right temporal ganglioglioma A: motor fMRI; B) 3D-SPGR; C: motor fMRI (red) fused with DT-tractography (yellow) (see text).

\section{Case 3 (Figure 4)}

This case illustrates the usefulness of fMRI in identifying the Wernicke's speech area in a 34 year old, left handed female with a right posterior temporal oligodendroglioma WHO II/IV. The fMRI located the Wernicke's area in the right posterior temporal lobe. Its location could be confirmed by intraoperative ECS (Figure 4, tracked cortical stimulator in yellow, speech activation in red). A subtotal iMRI-guided tumor resection was achieved and the patient left the hospital three days post-surgery without neurologic deficits.

\section{Discussion}

Our, as well as and other groups' [13] preliminary experience with diffusion tensor imaging suggests that functioning white matter fiber tracts can be present within intrinsic brain tumors. In our small patient cohort, two different tractography methods were used: the 3D-tractography method described by Westin [12] and the 2Dvisualization method described by Peled [14]. The results obtained with both methods were consistent (Figure 3).

In the case of left frontal oligodendroglioma presented above (illustrative case 1), the DT-MRI and fMRI identified motor fibers descending from the supplementary motor area through the anterior part of the tumor. In order to maximize tumor removal, it was decided to include the anterior part of the tumor in the resection. Post- 
surgery, the patient developed a typical SMA-syndrome. This fact suggests that functional motor fibers may have been present within the tumor. Consistency between fMRI and DTI data is an additional means for verifying the accuracy of fiber tract mapping.

On the conventional MRI of illustrative case 2, the tumor seems to displace the right cerebral peduncle and internal capsule (Figure 2). Nevertheless, the tractography clearly demonstrates tumor infiltration of the cortico-spinal tract (Figure 2). This example illustrates the limitations of conventional MRI in describing white matter anatomy.

FMRI data has proved to be a useful complement to intraoperative electro-cortical stimulation. In the illustrative case 3, preoperative fMRI located the sensory speech area in the right posterior temporal lobe and served as guide for intraoperative cortical stimulation and subsequent resection of a lesion assessed as being inoperable at another institution.

In order to maintain the accuracy of preoperative fMRI and DTI data throughout the surgical procedure, a mechanism for compensating brain shift changes needs to be put in place. In a previous study [7], we have shown that accurate non-rigid registration of preoperative data can be accomplished with our biomechanical simulation method within the time constraints imposed by the ongoing surgery. The tests we have conducted in this small patient population have proven our non-rigid registration method to be practicable also with more complex data sets, such as fMRI and DTI.

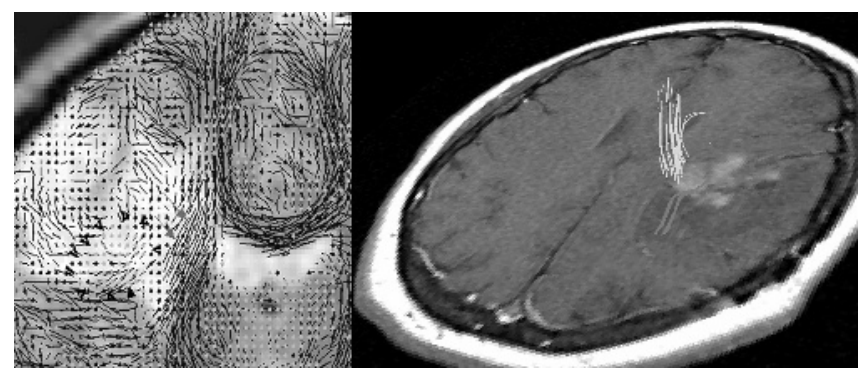

Fig. 3. Right fronto-temporal anaplastic astrocytoma. Left: red arrows: cortico-spinal tract; black arrow heads: arcuate fasciculus. Right: motor (yellow) and arcuate fasciculus (blue) fibers passing through contrast enhancing tumor area.

\section{Conclusion}

Although conventional (anatomical) MRI provides excellent definition of cortical surface, ventricular system, subcortical gray matter and intracranial pathology, it fails to identify functionally important cortical areas and white matter tracts and their relationship with the tumor. Such information, however, is of paramount importance for surgical planning and guidance. With the advent of fMRI and DTI, obtaining such information in advance of surgery became possible.

The changes in brain shape which occur during surgery, however, limit the use of preoperative imaging for real-time surgical guidance. Intraoperative re-acquisition of fMRI and DTI is impractical at present due to long image acquisition and processing 
times. The use of robust and fast biomechanical simulations for the purpose of preserving the accuracy of preoperative fMRI and DTI data appears to be a viable solution to the problem of brain shift.

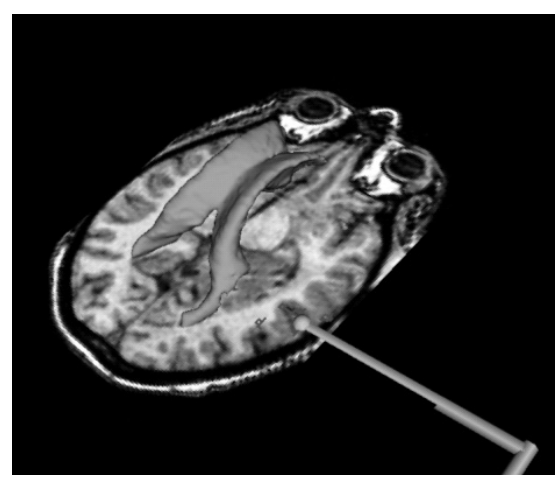

Fig. 4. (case 3). Wernicke's area (red) in a case of right posterior temporal oligodendroglioma WHO II/IV (see text).

\section{References}

1. Kelles GE, LK, Berger MS, Low-grade hemispheric gliomas in adults: a critical review of extent of resection as a factor influencing outcome. JNeurosurg, 2001. 95(5): p. 735-745.

2. Nabavi A, BP, Gering DT, Westin CF, Mehta V, Pergolizzi RS, Ferrant M, Warfield SK, Hata N, Schwartz RB, Wells WM III, Kikinis R, Jolesz F, Serial Intraoperative Magnetic Resonance Imaging of Brain Shift. Neurosurgery, 2001. 48(4): p. 787-798.

3. Black PM, Alexander E et al., Craniotomy for tumor treatment in an intraoperative magnetic resonance imaging unit. Neurosurgery, 1999. 45(3): p. 423-433.

4. Roux F-E, ID, Tremoulet M, et al., Methodological and technical issues for integrating functional magnetic resonance imaging data in a neuronavigational system. Neurosurgery, 2001. 49(5): p. 1145-1157.

5. Schiffbauer H, Ferari $\mathrm{P}$ et al., Functional activity within brain tumors: a magnetic source imaging study. Neurosurgery, 2001. 49(6): p. 1313-1321.

6. Ojemann, JG, Miller, JW et al., Preserved function in brain invaded by tumor. Neurosurgery, 1996. 39(2): p. 253-8; discussion 258-9.

7. Warfield, $\mathrm{S}, \mathrm{Talos}, \mathrm{F}$ et al. Real-time registration of volumetric brain MRI by biomechanical simulation of deformation during image guided neurosurgery. Comput Visual Sci, 2002. 5: p. 3-11.

8. Gering, DT, Nabavi, A et al., An integrated visualization system for surgical planning and guidance using image fusion and an open MR. J Magn Reson Imaging, 2001. 13(6): p. 967-75.

9. Wells WM III, VP, Atsumi, Nakajima S, Kikinis R, Multi-modal Volume Registration by Maximization of Mutual Information. Med Image Anal, 1996. 1(1): p. 35-51.

10. Peled, $\mathrm{S}, \mathrm{Gudbjartsson,} \mathrm{H}$ et al., Magnetic resonance imaging shows orientation and asymmetry of white matter fiber tracts. Brain Res, 1998. 780(1): p. 27-33.

11. Basser, PJ, Pajevic, S et al., In vivo fiber tractography using DT-MRI data. Magn Reson Med, 2000. 44(4): p. 625-32. 
12. Westin, CF, Maier, SE et al., Processing and visualization for diffusion tensor MRI. Med Image Anal, 2002. 6(2): p. 93-108.

13. Witwer, BP, Moftakhar, R et al., Diffusion-tensor imaging of white matter tracts in patients with cerebral neoplasm. J Neurosurg, 2002. 97(3): p. 568-75.

14. Peled S, HG, Westin C-F et al. Magnetic resonance imaging shows orientation and asymmetry of white matter fiber tracts. Brain Research, 1998. 780: p. 27-33. 